\title{
Review of: "The significance of pyrogenic polycyclic aromatic hydrocarbons in Borneo peat core for the reconstruction of fire history"
}

\author{
Xiaodong $\mathrm{Li}^{1}$ \\ 1 Zhejiang University
}

Potential competing interests: The author(s) declared that no potential competing interests exist.

It is intersting and meaningful to study the footprint of pyrogenic polycyclic aromatic hydrocarbons in the volcano case. However, this study was not enough for reconstructing the fire history. The result "the LMW PAHs could be transported from remote locations" should be further investigated. 